\title{
Prey size reconstruction based on myctophid otoliths in scats of northern fur seals (Callorhinus ursinus)
}

\author{
Shiroh Yonezaki ${ }^{1, *}$, Masashi Kiyota ${ }^{1}$, Norihisa Baba ${ }^{2}$, Takashi Koido ${ }^{3}$ and Akira Takemura ${ }^{3}$ \\ ${ }^{1}$ National Research Institute of Far Seas Fisheries, Fisheries Research Agency, Yokohama 236-8648, Japan \\ ${ }^{2}$ Seikai National Fisheries Research Institute, Fisheries Research Agency, Nagasaki 851-2213, Japan \\ ${ }^{3}$ Faculty of Fisheries, Nagasaki University, Nagasaki 852-8521, Japan
}

Diet studies of eared seals (Otariidae) are very important, because they are high-level consumers in marine ecosystems (Bowen 1997). Recently, non-lethal methods such as scat analysis have been applied to diet studies of eared seals (e.g., Zeppelin and Ream 2006; Sigler et al. 2009). In this analysis, diet compositions are estimated from the undigested hard part remains of prey items (fish otoliths, squid beaks, crustacean exoskeletons, etc.; Pierce and Boyle 1991). Fish species are identified based on the morphology of otoliths. Size and weight of fish consumed can also be estimated from the lengths of otoliths in scats (Sinclair et al. 1994). Although scat analysis is a useful method that provides dietary information without animal capture, the resultant information could include biases caused by digestion (Jobling and Breiby 1986; Yonezaki et al. 2003; Gudmundson et al. 2006). Fish otoliths are partially, or even completely, digested during passage through the alimentary system. Feeding experiments of various eared seals have been conducted to investigate the potential biasing effects of digestion on otoliths (e.g., Staniland 2002; Tollit et al. 2003). Numerical loss and length reduction rates of fish otoliths in scat samples were assessed from captive experiments, and correction factors calculated to enable the reconstruction of the original number and body lengths of fishes that were consumed. These correction factors are known to vary with prey species, eared seal species and experimental conditions (e.g., Bowen 2000; Orr and Harvey 2001; Staniland 2002). However, few studies have applied the correction factors to wild samples and tested the validity of the diet reconstruction.

The purpose of this study was to use experimentally designed meals and captive northern fur seals (Callorhinus ursinus) to calculate correction factors (CFs) for reconstruction of the myctophid fish size to compensate for the effects of digestion. Moreover, we apply the CFs to otoliths recovered from the large intestine contents of wild fur seal specimens, and estimated the size of the myctophid fish eaten. Myctophid fish used in the experiment are dominant components of micronekton communities in oceanic ecosystems and are important prey items of northern fur seals in the western North Pacific Ocean (Yonezaki et al. 2003; Yonezaki et al. 2008). Therefore, the calculation of CFs of myctophid fish otoliths enables data for use in the reconstruction of the diet of northern fur seals based on scats.

\section{Materials and methods}

\section{Feeding experiments}

Feeding experiments were conducted with a sub-adult male (NFS 1; 5-yr-old, body mass $41.5 \mathrm{~kg}$ ) and an adult female (NFS 2; 14-yr-old, body mass $30.6 \mathrm{~kg}$ ) northern fur seals kept in the Izu-Mito Sea Paradise (Aquarium), Shizuoka, Japan from November to December 2000. These experimental animals were kept in $8.0 \mathrm{~m}^{2}$ and 5.5 $\mathrm{m}^{2}$ compartments, respectively. They were maintained on a diet of blue mackerel (Scomber australasicus) and were fed twice daily (in the morning and evening) before each experimental period. Meal sizes were approximately $4.0 \mathrm{~kg} \mathrm{day}^{-1}$ (NFS 1) and $2.0 \mathrm{~kg} \mathrm{day}^{-1}$ (NFS 2).

The experimental prey (Japanese lanternfish Notoscopelus japonicus (JL) and California headlightfish Diaphus theta $(\mathrm{CH})$ ) were used from samples caught during a trawl survey off the Sanriku coast in August 2000. The long diameter length of sagittal otolith (OL, x: $\mathrm{mm})-$ standard length (SL, y: $\mathrm{cm}$ ) regression formula for JL was determined by the least-squares method using subsamples from the trawl survey in 2000. Regression formula for $\mathrm{CH}$ was used from Ohizumi et al. (2001). Regression formulae are given in the following equation:

*To whom correspondence should be addressed.E-mail: yonez@affrc.go.jp 
Japanese lanternfish:

$$
\mathrm{y}=1.789 \mathrm{x}+2.213\left(n=65, \mathrm{r}^{2}=0.72\right)
$$

California headlightfish:

$$
\mathrm{y}=2.964 \mathrm{x}-1.283 \text { (Ohizumi et al. 2001, } n=49, \mathrm{r}^{2}=0.84 \text { ) }
$$

The protocol of a feeding trial was that the fur seals were fed the experimental prey in the morning of the first day, after the experimental feeding, the fur seals were fed blue mackerel without heads and digestive organs twice a day until the end of each trial to avoid contamination of non-experimental otoliths. SLs of experimental prey (True-SLs) were measured to the nearest $0.1 \mathrm{~cm}$ prior to the experimental feeding. After experimental feeding, each animal was checked hourly during the day and scats were collected when they were observed. The animals were videotape-recorded during the night and scats were collected the following morning. The trial ended when otoliths no longer appeared in two successive scats. As a result, the duration of the trials was five days. During each trial fur seals were held on land, but were washed by water at the time of room cleaning in the morning and at feeding time. Fur seals were allowed to swim in pools between the trials.

Recovered scats were collected in plastic bags and soaked in the home detergent diluted with water. Scat samples were gently washed under running water through a series of three sieves $(2.0,1.0$, and $0.5 \mathrm{~mm})$ in the laboratory. All particles remaining on the sieves were collected and preserved in $70 \%$ ethanol. OLs were measured to the nearest $0.01 \mathrm{~mm}$ under a binocular microscope. Left and right otoliths were distinguished wherever possible.

For each feeding trial, recovery rates of otoliths were determined. SLs of JL and CH (Estimated-SL) were calculated from the recovered OLs using the above OL-
SL regression formulae.

The shapes of all the collected otoliths from scats can be identified to the prey species (Ohizumi et al. 2001). Therefore, CFs were calculated by dividing the mean True-SL by the mean Estimated-SL for each prey species and for male and female fur seals (Orr and Harvey 2001).

\section{Analysis of wild northern fur seals samples}

Ten northern fur seals (one juvenile male, one juvenile female, and eight adult females) were collected off the Sanriku coast $\left(37^{\circ} 18^{\prime} \mathrm{N}, 141^{\circ} 59^{\prime} \mathrm{E}\right)$ by R/V No.38 Kankimaru (413 GT) on 20 April 1998, as part of ongoing research monitoring program on the reproductive condition of this species. Their mean $( \pm S D)$ body weight and length were $33.5 \mathrm{~kg}( \pm 9.8)$. The fur seals were dissected onboard the research vessel, and the large intestines of specimens were preserved frozen at $-20^{\circ} \mathrm{C}$. The large intestine samples were thawed and incised later in the laboratory. Otoliths collected from the large intestine contents were treated using the same method as in the feeding experiments. Otoliths of myctophid fishes were identified based on the description of Ohizumi et al. (2001) and counted under a binocular microscope. These OLs were measured to the nearest $0.01 \mathrm{~mm}$. SLs were calculated using the regression formulae. Furthermore, the calculated SLs were corrected using the CFs determined from the feeding experiments. Considering that northern fur seals show significant sexual dimorphism, the CFs were applied to each sex.

\section{Trawl sampling and comparison of fish size composition}

Fish samples were collected during the night of 20 April 1998 off the Sanriku coast $\left(36^{\circ} 58^{\prime} \mathrm{N}, 142^{\circ} 10^{\prime} \mathrm{E}\right)$ with a trawl net towed at a speed of approximately 5 knots for 30 min by R/V Shunyo-maru (396 GT). Sam-

Table 1. Number $(n)$, standard length (SL $[\mathrm{cm}]$; mean and standard deviation $[S D]$ ) of experimental prey fed to northern fur seals. Recovery rates of otoliths in scats and calculated SLs from the recovered OLs using the OL-SL regression formula. Percentage of SL reduction calculated by

\begin{tabular}{|c|c|c|c|c|c|c|c|c|c|c|c|}
\hline \multicolumn{2}{|c|}{ Experimental prey species } & \multirow{3}{*}{ Trial code } & \multirow{3}{*}{$\begin{array}{l}\text { \% Recovery } \\
\text { of otoliths }{ }^{\#}\end{array}$} & \multicolumn{3}{|c|}{ Ingested prey species } & \multicolumn{3}{|c|}{$\begin{array}{l}\text { Estimated prey species } \\
\text { from otoliths in scats }\end{array}$} & \multirow{3}{*}{$\begin{array}{l}\% \mathrm{SL} \\
\text { reduction }\end{array}$} & \multirow{3}{*}{$\begin{array}{l}\text { Correction } \\
\text { factor }\end{array}$} \\
\hline \multirow{2}{*}{ Scientific name } & \multirow{2}{*}{ Common name } & & & \multirow{2}{*}{$n$} & \multicolumn{2}{|c|}{$\mathrm{SL}(\mathrm{cm})$} & \multirow{2}{*}{$n$} & \multicolumn{2}{|c|}{ SL $(\mathrm{cm})$} & & \\
\hline & & & & & Mean & $S D$ & & Mean & $S D$ & & \\
\hline \multirow{2}{*}{$\begin{array}{c}\text { Notoscopelus } \\
\text { japonicus }\end{array}$} & \multirow{2}{*}{$\begin{array}{l}\text { Japanese } \\
\text { lanternfish }\end{array}$} & JL-NFS 1 & 90.3 & 36 & 13.2 & 0.8 & 65 & 10.8 & 1.1 & $18.2^{*}$ & 1.22 \\
\hline & & JL-NFS 2 & 54.1 & 37 & 13.0 & 0.9 & 40 & 9.2 & 1.7 & $29.2^{*}$ & 1.41 \\
\hline \multirow[t]{2}{*}{ Diaphus theta } & \multirow{2}{*}{$\begin{array}{l}\text { California } \\
\text { headlightfish }\end{array}$} & CH-NFS 1 & 85.5 & 100 & 6.8 & 1.1 & 170 & 6.2 & 1.3 & $8.8^{*}$ & 1.10 \\
\hline & & CH-NFS 2 & 88.0 & 100 & 6.7 & 1.1 & 176 & 5.8 & 1.4 & $13.4^{*}$ & 1.15 \\
\hline
\end{tabular}
the comparison of True-SL and Estimated-SL and the correction factors for SL.

\# \% Recovery $=($ Left otoliths + Right otoliths + the total number of otoliths of undetermined orientation $) / 2 \times n \times 100$

* significant difference $(P<0.01)$ 
pling depths ranged from 0 to $50 \mathrm{~m}$ (1st trawl, $0-25 \mathrm{~m}$, 2nd trawl, 25-50 m) to cover the main feeding depths of northern fur seals (Gentry et al. 1986). The trawl net had a opening of $30 \mathrm{~m} \times 30 \mathrm{~m}$, total length of $90 \mathrm{~m}$, and $10 \mathrm{~mm}$ meshed cod-end. Trawl samples were sorted by species on deck. Subsamples of JL and $\mathrm{CH}$ were extracted at random from the trawl samples, and SLs were measured to the nearest $0.1 \mathrm{~cm}$. $\mathrm{JH}$ and $\mathrm{CH}$ were mainly collected from the first (sampling depth, 0-25 m) and second trawls (sampling depth, 25-50 m), respectively.

The SL compositions estimated from OLs of JL and $\mathrm{CH}$ collected from the large intestine contents of fur seals with and without the correction by using the CFs of different sexes that were compared with the SL compositions of the trawl samples. All statistical analyses were performed using R. 2.9.2 (R Development Core Team, http://www.r-project.org/).

\section{Results and discussion}

Six scats (JL-NFS 1), eight scats (JL-NFS 2) and four samples of regurgitated materials (JL-NFS 2) were collected in the JL experiments. Five scats (CH-NFS 1), seven scats (CH-NFS 2) and one sample of regurgitated material (CH-NFS 1), one sample of regurgitated material (CH-NFS 2) were collected in the $\mathrm{CH}$ experiments. All collected otoliths were recovered from scats; the otoliths did not appear in the samples of regurgitated materials. This is because all OLs were smaller than the diameter of the pyloric end of the stomach of the fur seals (Yonezaki et al. 2003). Most of the otoliths were recovered from scats excreted within two days after feeding (Fig. 1). The excretion pattern was similar to other captive feeding experiments of California sea lions (Zalophus californianus) (Orr and Harvey 2001).

The recovery rates of otoliths from scats were high (54.1-90.3\%) despite the small sizes of myctophid otoliths (OL range: 1.75-6.21 mm) (Table 1). In other feeding experiments using otariids (California sea lion and South American fur seal Arctocephalus australis), the recovery rates of otoliths that were approximately the same size as the myctophid otoliths were low (26.449.4\%) (Dellinger and Trillmich 1988). In general, myctophid otoliths are large for their body sizes and robust (Ohizumi et al. 2001). The high recovery rates of our experiments are related to the characteristics of the myctophid otoliths.

The mean OLs of JL and CH collected from scats were
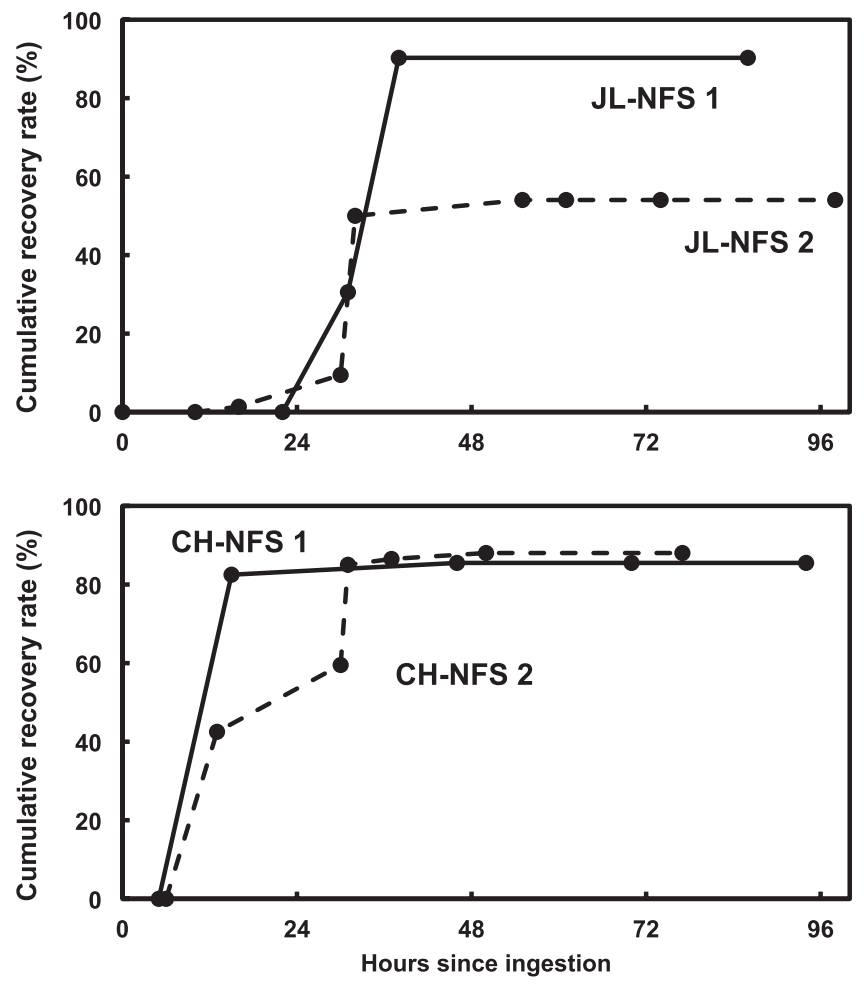

Fig. 1. Cumulative percentages of myctophid otoliths recovered from scats from two northern fur seals after the time of ingestion during the captive feeding experiments.

$4.80 \mathrm{~mm}( \pm 0.61)(\mathrm{JL}-\mathrm{NFS} 1), 3.89 \mathrm{~mm}( \pm 0.97)(\mathrm{JL}-\mathrm{NFS}$ 2), $2.52 \mathrm{~mm}( \pm 0.44)(\mathrm{CH}-\mathrm{NFS} 1)$, and $2.38 \mathrm{~mm}( \pm 0.47)$ (CH-NFS 2). For both individuals the mean EstimatedSLs were significantly smaller than the True-SLs (JLNFS 1: $F$-test: $F=1.7406, P=0.07723, t$-test: $t=$ $-11.7399, P<0.01$, JL-NFS 2: $F$-test: $F=3.3879, P=$ 0.0003546 , Welch Two Sample $t$-test: $t=-12.2781, P<$ $0.01, \mathrm{CH}-\mathrm{NFS} 1: F$-test: $F=1.3139, P=0.1371, t$-test: $t=-3.8162, P<0.01, \mathrm{CH}-\mathrm{NFS} 2: F$-test: $F=1.5767, P=$ 0.01325 , Welch Two Sample $t$-test: $t=-6.3339, P<$ $0.01)$.

337 otoliths (JL) and 38 otoliths $(\mathrm{CH})$ were collected from the large intestine contents of the wild fur seals.

The mean SLs of JL and $\mathrm{CH}$ of reconstructed without CFs were significantly $0.92(\mathrm{JL})$ and $0.94(\mathrm{CH})$ times more than mean SLs of the trawl sample (JL: $F$-test: $F=1.6863, P=0.02451$, Welch Two Sample $t$-test: $t=-10.4519, P<0.01, \mathrm{CH}: F$-test: $F=3.4644, P<$ 0.01 , Welch Two Sample $t$-test: $t=-2.8467, P<0.01$ ). The factor of the underestimation can be considered the effects of digestion. However, if we use the CFs derived from the captive experiments, the mean reconstructed SLs were significantly $1.29(\mathrm{JL})$ and $1.07(\mathrm{CH})$ 
Table 2. Mean estimated SLs with and without using CFs (SL [cm]; mean and standard deviation [SD]) from Notoscopelus japonicus and Diaphus theta otoliths collected from large intestine contents of fur seals. Mean SLs of $N$. japonicus and D. theta collected by trawl net sampling.

\begin{tabular}{|c|c|c|c|c|c|c|c|c|c|c|}
\hline \multicolumn{2}{|c|}{ Prey species } & \multicolumn{3}{|c|}{ Without CF } & \multicolumn{3}{|c|}{ With CF } & \multicolumn{3}{|c|}{ Trawl sample } \\
\hline Scientific name & Common name & $n$ & Mean & $S D$ & $n$ & Mean & $S D$ & $n$ & Mean & $S D$ \\
\hline Notoscopelus japonicus & Japanese lanternfish & 337 & 11.5 & 0.8 & 337 & 16.1 & 1.2 & 52 & 12.5 & 0.6 \\
\hline Diaphus theta & California headlightfish & 38 & 6.7 & 0.9 & 38 & 7.6 & 1.0 & 41 & 7.1 & 0.5 \\
\hline
\end{tabular}
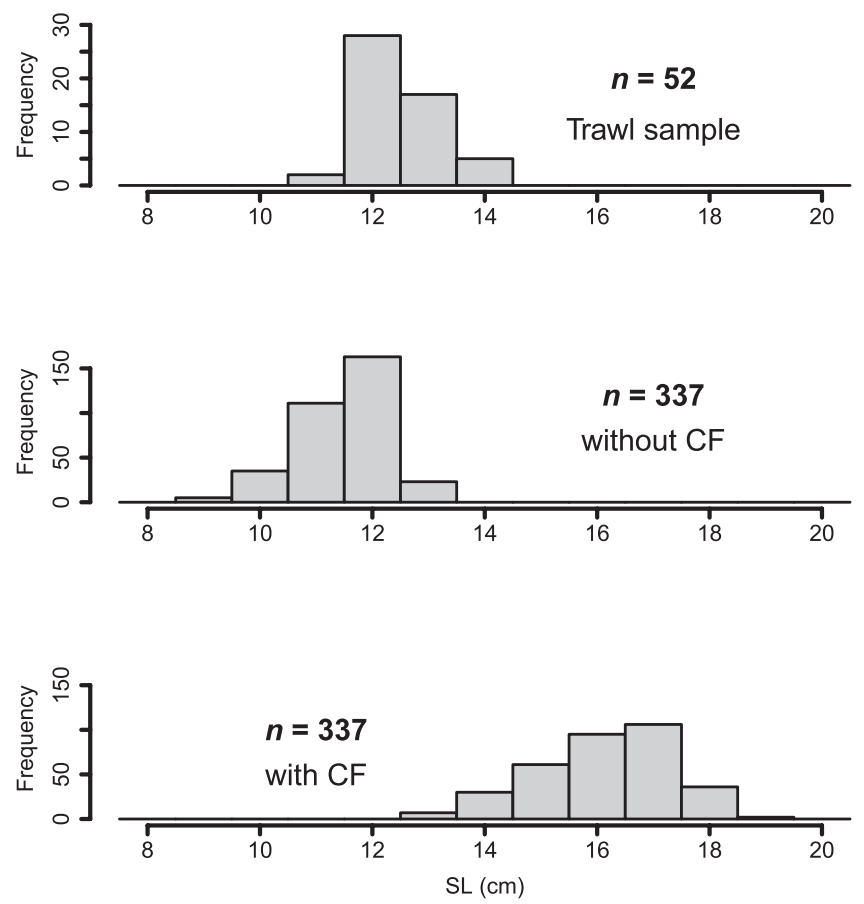

Fig. 2. Standard length (SL) composition of Japanese lanternfish collected by trawl net sampling (Upper). Estimated SL compositions (with and without correction) from Japanese lanternfish otoliths collected from large intestine contents of fur seals are shown in the middle and lower panels, respectively.

times more than that of the trawl samples (JL: $F$-test: $F=3.6331, P<0.01$, Welch Two Sample $t$-test: $t=$ 34.3224, $P<0.01, \mathrm{CH}: F$-test: $F=4.3103, P<0.01$, Welch Two Sample $t$-test: $t=2.92, P<0.01$ ) (Table 2 and Figs. 2 and 3). Three explanations are possible for this discrepancy in prey size: (i) size-selective feeding on myctophids by wild fur seals (ii) size-selective sampling in our trawl survey (iii) overestimation of SLs by using CFs derived from captive feeding experiments. There is only very limited information available in the literature on size-selective predation by northern fur seals. It may be difficult for fur seals to select large size fishes in the same patch while foraging, considering that the predation of myctophids by fur seals occurs at nighttime (Yonezaki et al. 2008). They capture prey species mainly depending on vision. In this study, the variances
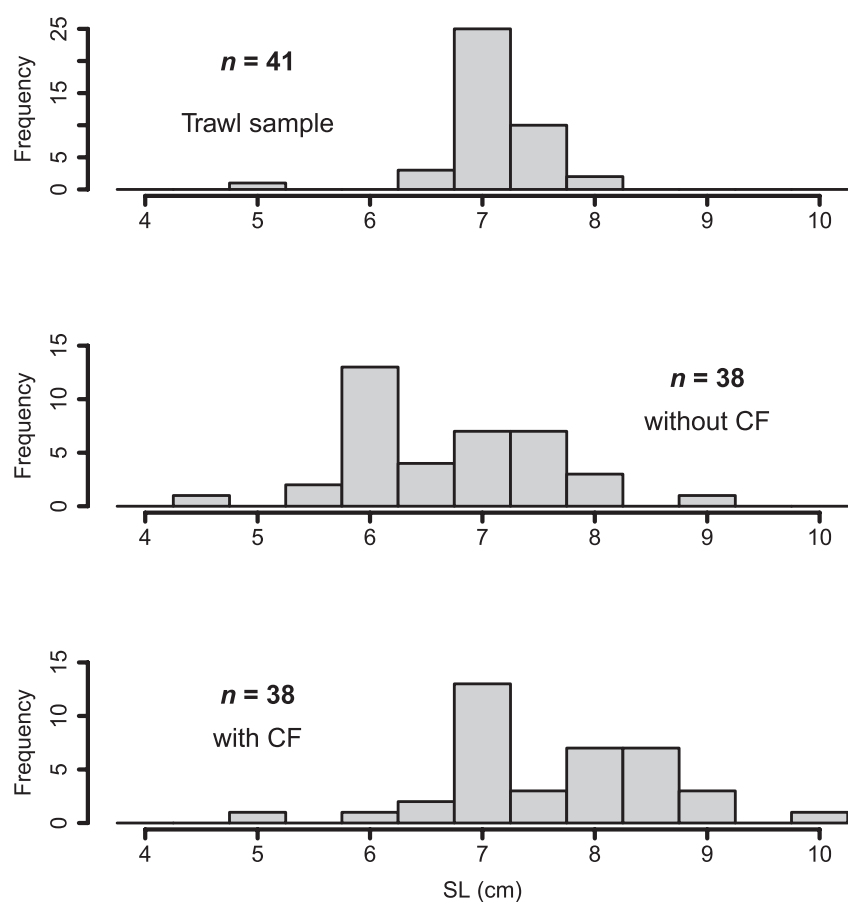

Fig. 3. Standard length (SL) compositions of California headlightfish collected by trawl net sampling (Upper). Estimated SL compositions (with and without correction) from California headlightfish otoliths collected from large intestine contents of fur seals are shown in the middle and lower panels, respectively.

of corrected SLs were significantly larger than the trawl samples. Prey size selectivity by fur seals could not explain the discrepancy in prey size.

There was a report that the small sized fishes of myctophid patch were distributed at shallow water depth (Yatsu et al. 2005). However, the difference in the size composition was at the level of a few millimeters. Considering the swimming speed of myctophid fishes and towing speed of trawl sampling, it is hard to account for smaller fishes being sampled selectively.

Under experimental conditions, depending on the level of animal activity, the excretion rate differs (Tollit et al. 2003). In the inactive condition, fish otoliths remain in the stomach for a longer time, and therefore become more susceptible to digestion by digestive acids. Therefore, the otoliths will be more reduced. On the 
other hand, in the wild, the so-called active condition, otoliths do not remain in the stomach and are not exposed to the effects of digestive acids for as long as inactive seals (Dellinger and Trillmich 1988). Therefore if CF calculated under experimental conditions is applied to fur seals in the wild, it would overestimate the prey size. Differences in SL compositions between prey of wild fur seals with CFs and trawl samples are considered to be due to the above overestimate. In this study, (i) and (ii) hypotheses can not be excluded completely, but in order to calculate the CF based on feeding experiments, seals in the active condition should be assessed.

Acknowledgments: We thank Tadao Tonomori, Kenji Maejima, Kaoru Kohyama, Noriyasu Suzuki, and other staff of Izu-Mito Sea Paradise (Aquarium) for their help with the feeding experiments. We thank the crew of the R/V Shunyo-maru and No.38 Kanki-maru for their cooperation and assistance for the sample collection at sea. This study was conducted under the fur seal research permit issued by the Ministry of Agriculture, Forestry and Fisheries, Japan.

\section{References}

Bowen, W. D. 1997. Role of marine mammals in aquatic ecosystems. Marine Ecology Progress Series 158: 267-274.

Bowen, W. D. 2000. Reconstruction of pinniped diets: Accounting for complete digestion of otoliths and cephalopod beaks. Canadian Journal of Fisheries and Aquatic Sciences 57: 898-905.

Dellinger, T. and Trillmich, F. 1988. Estimating diet composition from scat analysis in otariid seals (Otariidae): Is it reliable? Canadian Journal of Zoology 66: 1865-1870.

Gentry, R. L., Kooyman, G. L. and Goebel, M. E. 1986. Feeding and diving behavior of northern fur seals. In (R. L. Gentry and G. L. Kooyman, eds.) Fur Seals: Maternal Strategies on Land and at Sea, pp. 61-78. Princeton University Press.

Gudmundson, C. J., Zeppelin, T. K. and Ream, R. R. 2006. Application of two methods for determining diet of northern fur seals (Callorhinus ursinus). Fishery Bulletin 104: 445-455.

Jobling, M. and Breiby, A. 1986. The use and abuse of fish otoliths in studies of feeding habits of marine piscivores. Sarsia 71:265274.

Ohizumi, H., Watanabe, H., Moku, M. and Kawahara, S. 2001. Species identification for otoliths of myctophid fishes in the western North Pacific. Aquabiology 137: 626-637 (in Japanese with English abstract).

Orr, A. J. and Harvey, J. T. 2001. Quantifying errors associated with using fecal samples to determine the diet of the California sea lion (Zalophus californianus). Canadian Journal of Zoology 79: 1080-1087.

Pierce, G. J. and Boyle, P. R. 1991. A review of methods for diet analysis in piscivorous marine mammals. Oceanography and Marine Biology: An Annual Review 29: 409-486.

Sigler, M. F., Tollit, D. J., Vollenweider, J. J., Thedinga, J. F., Csepp, D. J., Womble, J. N., Wong, M. A., Rehberg, M. J. and Trites, A. W. 2009. Steller sea lion foraging response to seasonal changes in prey availability. Marine Ecology Progress Series 388: 243 261.

Sinclair, E., Loughlin, T. and Pearcy, W. 1994. Prey selection by northern fur seals (Callorhinus ursinus) in the eastern Bering Sea. Fishery Bulletin 92: 144-156.

Staniland, I. J. 2002. Investigating the biases in the use of hard prey remains to identify diet composition using Antarctic fur seals (Arctocephalus gazella) in captive feeding trials. Marine Mammal Science 18: 223-243.

Tollit, D. J., Wong, M., Winship, A. J., Rosen, D. A. S. and Trites, A. W. 2003. Quantifying errors associated with using prey skeletal structures from fecal samples to determine the diet of Steller's sea lion (Eumetopias jubatus). Marine Mammal Science 19: 724-744.

Yatsu, A., Sassa, C., Moku, M. and Kinoshita, T. 2005. Night-time vertical distribution and abundance of small epipelagic and mesopelagic fishes in the upper $100 \mathrm{~m}$ layer of the KuroshioOyashio transition zone in spring. Fisheries Science 71: 12801286.

Yonezaki, S., Kiyota, M. and Baba, N. 2008. Decadal changes in the diet of northern fur seal (Callorhinus ursinus) migrating off the Pacific coast of northeastern Japan. Fisheries Oceanography 17: 231-238.

Yonezaki, S., Kiyota, M., Baba, N., Koido, T. and Takemura, A. 2003. Size distribution of the hard remains of prey in the digestive tract of northern fur seal (Callorhinus ursinus) and related biases in diet estimation by scat analysis. Mammal Study 28: 97-102.

Zeppelin, T. K. and Ream, R. R. 2006. Foraging habitats based on the diet of female northern fur seals (Callorhinus ursinus) on the Pribilof Islands, Alaska. Journal of Zoology 270: 565-576.

Received 13 June 2010. Accepted 7 April 2011. 